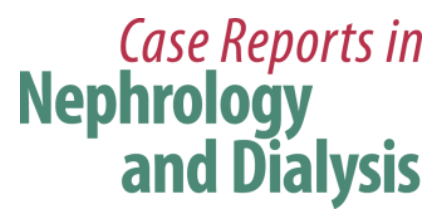

Case Rep Nephrol Dial 2020;10:9-17

DOI: $10.1159 / 000506118$

Published online: February 12, 2020

(c) 2020 The Author(s)

Published by S. Karger AG, Basel

www.karger.com/cnd

This article is licensed under the Creative Commons Attribution-NonCommercial 4.0 International License (CC BY-NC) (http://www.karger.com/Services/OpenAccessLicense). Usage and distribution for commercial purposes requires written permission.

\title{
Long-Term Effects of High-Dose Tolvaptan for Autosomal Dominant Polycystic Kidney Disease Patients
}

\author{
Shinya Nakatani ${ }^{a} \quad$ Eiji Ishimura ${ }^{b} \quad$ Yuri Machiba $^{a} \quad$ Kenta Fujimoto $^{a}$ \\ Hideki Uedono ${ }^{a}$ Akihiro Tsuda ${ }^{a}$ Katsuhito Mori ${ }^{b}$ Masanori Emoto ${ }^{a}$ \\ Masaaki Inabaa, b \\ aDepartment of Metabolism, Endocrinology, and Molecular Medicine, Osaka City \\ University Graduate School of Medicine, Osaka, Japan; 'bepartment of Nephrology, \\ Osaka City University Graduate School of Medicine, Osaka, Japan
}

\section{Keywords}

Autosomal dominant polycystic kidney disease $\cdot$ Tolvaptan $\cdot$ High dose $\cdot$ Long term

\section{Abstract}

Tolvaptan, a vasopressin $V_{2}$ receptor antagonist, was initially approved in Japan for treatment of autosomal dominant polycystic kidney disease (ADPKD). Recently, a retrospective study showed that the effect of tolvaptan on kidney function could be sustained for a long period. However, the long-term efficacy and safety of high-dose tolvaptan $(120 \mathrm{mg} /$ day $)$ in individual cases remain unknown. We report here 2 Japanese ADPKD patients (males, 36 and 29 years old) treated with tolvaptan ( $120 \mathrm{mg} /$ day) for 9 years, during which time determinations of estimated glomerular filtration rate (eGFR) and total kidney volume (TKV) were performed. In these 2 patients, eGFR prior to therapy was 57.3 and $76.3 \mathrm{~mL} / \mathrm{min} / 1.73 \mathrm{~m}^{2}$, respectively, and 30.2 and $43.5 \mathrm{~mL} / \mathrm{min} / 1.73 \mathrm{~m}^{2}$, respectively, after 9 years of tolvaptan treatment, for a relatively constant annual decline of -3.01 and $-3.64 \mathrm{~mL} / \mathrm{min} / 1.73 \mathrm{~m}^{2}$, respectively. As compared to the predicted (calculated) eGFR without tolvaptan treatment, eGFR actually measured was higher 


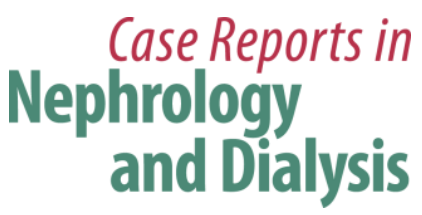

Case Rep Nephrol Dial 2020;10:9-17

by 15.3 and $12.6 \mathrm{~mL} / \mathrm{min} / 1.73 \mathrm{~m}^{2}$, respectively, after the 9-year therapy period. In addition, the rate of TKV increase was gradual, 2.4 and $4.7 \%$, respectively, per year during the initial 3-year period, to 6.5 and $12.5 \%$, respectively, per year in the following 6-year period. During the 9 years of treatment, neither patient showed tolvaptan-related adverse events. Our findings suggest that long-term administration of tolvaptan at a high dose is both safe and effective to preserve kidney function, though a gradual increase in TKV was seen in both of the present cases, particularly during the later phase.

(C) 2020 The Author(s)

Published by S. Karger AG, Basel

\section{Introduction}

Autosomal dominant polycystic kidney disease (ADPKD), the most common genetic cause of chronic kidney disease (CKD) worldwide, results in progressive development of kidney cysts, increased kidney volume, kidney pain, hypertension, gross hematuria, and ultimately kidney failure [1]. In Japan, approximately 1,000 cases of end stage renal disease (ESRD) annually are attributable to ADPKD, and it is the fourth leading cause of ESRD, following diabetes, glomerulonephritis, and hypertension [2].

The antidiuretic hormone arginine vasopressin and its second messenger cAMP have been identified as promoters of cellular proliferation of cysts and kidney enlargement in animal models of cystic kidney disease [3], while vasopressin $V_{2}$ receptor blockades have been shown to reduce cyst burden and protect kidney function in rodent models of cystic kidney disease [4]. As for cyst epithelial cells in humans, tolvaptan, a vasopressin $V_{2}$ receptor antagonist, has been reported to inhibit cell proliferation and chloride-dependent fluid secretion in a dose-dependent manner [5]. This drug was recently introduced for treatment of ADPKD patients, following the results of the Tolvaptan Efficacy and Safety in Management of Autosomal Dominant Polycystic Kidney Disease and Its Outcome (TEMPO) 3:4 trial [6]. The TEMPO 3:4 trial was performed as a randomized double-blind placebo-controlled parallel-group investigation, and results obtained over a 3-year period demonstrated that administration of tolvaptan reduced the annual rate of increase in total kidney volume (TKV) and lowered that of kidney function decline [6].

Based on the results of the TEMPO 3:4 trial, tolvaptan was initially approved by the regulatory authority in Japan in March 2014 as therapy for ADPKD patients with CKD stages 1-3, indicating rapidly progressive disease. Subsequently, the Replicating Evidence of Preserved Renal Function: an Investigation of Tolvaptan Safety and Efficacy in ADPKD (REPRISE) trial was conducted using ADPKD patients with a later CKD stage (estimated glomerular filtration rate [eGFR] 25-65 mL/min/1.73 $\mathrm{m}^{2}$ ) [7]. Results of the REPRISE trial showed a slower eGFR decline with tolvaptan as compared to a placebo. Thereafter, tolvaptan was approved by the United States Food and Drug Administration (FDA) (April 2018).

Recently, a retrospective study conducted at the Mayo Clinic in the United States showed that the effects of tolvaptan for protection of kidney function could be sustained for a long period ( $n=97$ patients, average follow-up 4.6 years) [8], though the effect on increasing TKV was not analyzed. Inhibition of cell proliferation and chloride-dependent fluid secretion by tolvaptan has been shown to occur in a dose-dependent manner, while its long-term efficacy and safety when given at a high dose remain unknown. Here, we present 2 Japanese ADPKD 


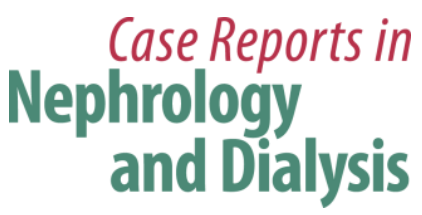

Case Rep Nephrol Dial 2020;10:9-17

patients who received high-dose tolvaptan therapy (total daily dose $120 \mathrm{mg}$ ) for a period of 9 years, since the beginning of the TEMPO 3:4 trial.

\section{Case Presentation}

\section{Case 1}

A 36-year-old male was diagnosed with ADPKD at the Outpatient Department of our hospital in 2008, based on magnetic resonance imaging (MRI) findings. Previously in 2002, he presented with gross hematuria. Family history related to ADPKD was unclear. The patient had hypertension and was being treated with antihypertensives including valsartan (40 $\mathrm{mg}$ /day). Blood pressure was 108/69 mm Hg. The case was complicated with multiple liver cysts, while no other complications were noted, such as brain aneurysm or heart valve disease. An MRI examination revealed enlargement of the bilateral kidneys with multiple cysts of various sizes, and TKV determined with an ellipsoid equation was 1,497 mL. Table 1 shows blood examination and urinalysis results prior to beginning treatment with tolvaptan.

Following an explanation of the procedures, the patient explicitly expressed a desire to participate in the TEMPO 3:4 trial. eGFR, calculated using the Japanese formula [9], was 57.3 $\mathrm{mL} / \mathrm{min} / 1.73 \mathrm{~m}^{2}$, and estimated creatinine clearance calculated with the Cockcroft-Gault formula [10] was $78.1 \mathrm{~mL} / \mathrm{min}$ (Table 1). Thus, the patient was considered eligible to be registered. Three years after beginning the trial, when the study had finished, the clinical research coordinator of Osaka City University Hospital revealed that the patient had been assigned to the tolvaptan group. According to the protocol of the TEMPO 3:4 trial [6], tolvaptan was started at a dose of $60 \mathrm{mg} /$ day (45 mg given in the morning, $15 \mathrm{mg}$ in the evening), with a weekly increase of $30 \mathrm{mg} /$ day until reaching $120 \mathrm{mg} /$ day (90 mg in the morning, $30 \mathrm{mg}$ in the evening).

The eGFR in this patient was $57.3 \mathrm{~mL} / \mathrm{min} / 1.73 \mathrm{~m}^{2}$ prior to therapy and 30.2 $\mathrm{mL} / \mathrm{min} / 1.73 \mathrm{~m}^{2}$ after 9 years of treatment with tolvaptan, for an annual decline of -3.01 $\mathrm{mL} / \mathrm{min} / 1.73 \mathrm{~m}^{2}$. Also, TKV was $1,497 \mathrm{~mL}$ before starting treatment and 2,328 $\mathrm{mL}$ after the 9-year treatment period, for an annual rate of increase of 6.2\% (Fig. 1). TKV showed a gradual increase, from $2.4 \%$ per year during the initial 3-year period to $6.5 \%$ per year in the most recent 6-year period. After several weeks of tolvaptan treatment, the patient showed no tolvaptan-related adverse events, such as liver dysfunction, hypernatremia, or polyuria, which were common adverse events noted in the TEMPO 3:4 trial tolvaptan group [6]. Currently, tolvaptan treatment is continuing and his condition remains stable. During the study period, blood pressure remained stable (under 130/80 $\mathrm{mm} \mathrm{Hg}$ ). However, 1 year after the end of the study, it had elevated to $145 / 86 \mathrm{~mm} \mathrm{Hg}$ and amlodipine at $2.5 \mathrm{mg}$ was administrated.

Case 2

A 29-year-old male with gross hematuria was diagnosed with ADPKD at the Outpatient Department of our hospital, based on MRI findings. There was a family history of ADPKD, and his father suffered from ESRD. Hypertension was also present, and the patient was being treated with antihypertensives including candesartan $(8 \mathrm{mg} /$ day $)$. Blood pressure was 128/76 mm Hg. Multiple liver cysts were evident, though no other complications, including brain aneurysm or heart valve disease, were noted. The MRI examination also revealed 


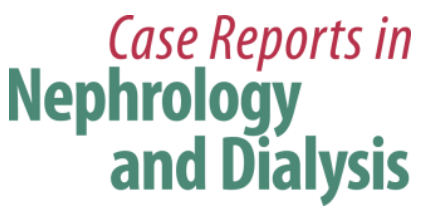

Case Rep Nephrol Dial 2020;10:9-17

enlargement of the bilateral kidneys with multiple cysts of various sizes, and TKV determined with an ellipsoid equation was 1,290 mL. Table 1 shows blood examination and urinalysis results prior to starting treatment with tolvaptan.

Serum creatinine was $0.97 \mathrm{mg} / \mathrm{dL}$, the eGFR, calculated by the Japanese formula [9], was $76.3 \mathrm{~mL} / \mathrm{min} / 1.73 \mathrm{~m}^{2}$, and estimated creatinine clearance, calculated with the CockcroftGault formula [10], was $125 \mathrm{~mL} / \mathrm{min}$ (Table 1). After obtaining informed consent, the patient was registered in the TEMPO 3:4 trial. Three years after beginning that, the clinical research coordinator of Osaka City University Hospital revealed that he had been assigned to the tolvaptan group. According to the protocol of the trial [6], tolvaptan was started at $60 \mathrm{mg} /$ day and then increased up to $120 \mathrm{mg} /$ day, the same as described above for case 1 .

After 3 years of tolvaptan treatment, blood pressure showed elevation and amlodipine at $10 \mathrm{mg} /$ day was added, which resulted in systolic and diastolic blood pressure values stabilizing at 132-146 and 72-86 $\mathrm{mm} \mathrm{Hg}$, respectively. At 5 years after beginning the administration of tolvaptan, blood pressure was elevated again, and the antihypertensive medication administration was changed to a combination tablet comprised of $80 \mathrm{mg}$ of valsartan and $10 \mathrm{mg}$ of cilnidipine, though blood pressure remained high at 150-170 mm $\mathrm{Hg}$. eGFR was 76.3 $\mathrm{mL} / \mathrm{min} / 1.73 \mathrm{~m}^{2}$ prior to starting tolvaptan therapy and reduced to $43.5 \mathrm{~mL} / \mathrm{min} / 1.73 \mathrm{~m}^{2}$ after 9 years of treatment, for a decline of $-3.64 \mathrm{~mL} / \mathrm{min} / 1.73 \mathrm{~m}^{2}$ per year. Furthermore, TKV determined with an ellipsoid equation increased from $1,290 \mathrm{~mL}$ before starting tolvaptan treatment to $2,570 \mathrm{~mL}$ at the most recent examination, for an annual rate of increase of $11.0 \%$ (Fig. 1B). TKV in case 2 showed a gradual increase, from $4.7 \%$ per year in the initial 3 -year period to $12.5 \%$ per year in the most recent 6-year period. Although renal cyst infection has occurred twice during the 9-year treatment period, other adverse events related to tolvaptan, such as liver dysfunction or hypernatremia, have not been seen in this patient. Currently, tolvaptan treatment is continuing and his condition remains stable.

\section{Discussion}

The efficacy of tolvaptan at a daily dose of 60-120 mg for ADPKD patients has been confirmed by results of 2 different pivotal trials [6, 7]. In a subgroup analysis conducted during the 3-year TEMPO 3:4 trial, the annual eGFR change was $-3.83 \mathrm{~mL} / \mathrm{min} / 1.73 \mathrm{~m}^{2}$ in Japanese patients [11]. In the present 2 cases, the annual eGFR decline during the longer 9 -year observation period was $-3.01 \mathrm{~mL} / \mathrm{min} / 1.73 \mathrm{~m}^{2}$ and $-3.64 \mathrm{~mL} / \mathrm{min} / 1.73 \mathrm{~m}^{2}$, respectively, similar to the Japanese subgroup analysis results shown in the TEMP03:4 trial [11]. Thus, high-dose tolvaptan treatment for protection of kidney function in Japanese ADPKD patients can be maintained for an extended period.

The beneficial effect of tolvaptan for ADPKD patients can be demonstrated by comparing observed (with tolvaptan treatment) and predicted (without tolvaptan treatment) eGFR values using a validated predictive equation [12]. Had tolvaptan treatment not been performed for the present 2 patients, eGFR after 9 years was predicted to be $14.9 \mathrm{~mL} / \mathrm{min} / 1.73 \mathrm{~m}^{2}$ in case 1 and $30.9 \mathrm{~mL} / \mathrm{min} / 1.73 \mathrm{~m}^{2}$ in case 2 . With that equation, the difference between observed and predicted eGFR in case 1 was calculated to be $15.3 \mathrm{~mL} / \mathrm{min} / 1.73 \mathrm{~m}^{2}$, while that in case 2 was calculated to be $12.6 \mathrm{~mL} / \mathrm{min} / 1.73 \mathrm{~m}^{2}$. These results show the benefit of long-term tolvaptan therapy for ADPKD. 


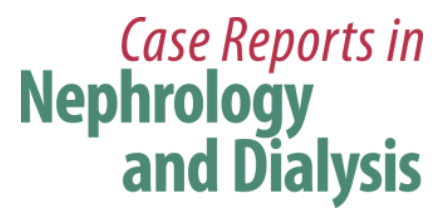

Case Rep Nephrol Dial 2020;10:9-17

A recent retrospective study conducted at the Mayo Clinic in the United States showed that ADPKD patients treated with tolvaptan had a lower eGFR slope from the baseline $(-2.20$ $\mathrm{mL} / \mathrm{min} / 1.73 \mathrm{~m}^{2}$ per year$)$ as compared with the control group $\left(-3.50 \mathrm{~mL} / \mathrm{min} / 1.73 \mathrm{~m}^{2}\right.$ per year) during the follow-up period (average 4.6 years) [8]. Although the details of each case were unclear in that study, the findings in the present patients obtained over a longer period were consistent with those of that study. Japan was the first country to approve tolvaptan for treatment of ADPKD, with more than 1,000 patients since treated, thus larger-scale and longer follow-up studies are needed to confirm the efficacy and safety of therapy for Japanese ADPKD patients.

During the 3-year TEMPO 3:4 trial, the annual rate of increase in TKV in the placebo group was $5.5 \%$, while that in the tolvaptan group was $2.8 \%$ [6]. In sub-analysis findings of Japanese patients, the annual rate of increase in TKV in the placebo group was 5.0\%, while that in the tolvaptan group was $1.3 \%$ [11]. During the initial 3-year treatment period of the present case 1, TKV increased from 1,497 to 1,606 mL, for an annual rate of increase of $2.4 \%$, similar to results shown in previous studies $[6,13]$. Notably, TKV in case 1 showed a gradual increase over time, from $2.4 \%$ per year in the initial 3 -year period to $6.5 \%$ per year during the most recent 6-year period. The increase in TKV growth in case 2 was also shown to be gradual, from $4.7 \%$ per year in the initial 3-year period to $12.5 \%$ per year in the most recent 6 -year period. The effect of tolvaptan on kidney volume in the early phase of ADPKD may be due to deflation caused by reduced fluid secretion from renal cysts, while that in the late phase is thought to be related to suppressed cell proliferation $[1,13]$. A recent retrospective study conducted at the Mayo Clinic in the United States did not evaluate TKV [8], thus this is the first case report in which evaluation of TKV over a long term was performed in ADPKD patients who received tolvaptan treatment. Considering the clinical courses of our patients, tolvaptan may be less effective when administered for more than 3-6 years, at least for inhibition of kidney volume enlargement. The exact mechanisms underlying an increase in TKV over the long term remain unknown. Studies of cases with longer follow-up periods are needed for confirmation of increased TKV.

Generally, increased TKV is associated with eGFR in ADPKD patients who do not undergo tolvaptan treatment [14]. That increase was not likely associated with eGFR decline in the present case 1, whereas it did seem to be at least somewhat associated with eGFR decline in case 2. Determination of TKV may not be appropriate to monitor the efficacy of tolvaptan. Indeed, a recently published review, which contains a practical guide for treatment of rapidly progressive ADPKD with tolvaptan, does not recommend annual TKV measurements to monitor efficacy of the therapy [15]. Further cohort studies of ADPKD patients who undergo tolvaptan treatment for a long period are needed to determine whether annual TKV measurements can predict eGFR decline and would also be informative in regard to other factors.

This is the first case report showing both long-term efficacy and safety of high-dose tolvaptan for protection of kidney function in Japanese patients with ADPKD. Results of the present 2 cases suggest that continued effectiveness with longer treatment may be reduced, at least in terms of kidney volume expansion. To confirm the effects of long-term therapy, it will be necessary to accumulate additional cases with longer periods of tolvaptan treatment. 


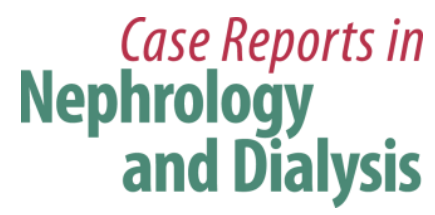

Case Rep Nephrol Dial 2020:10:9-17

DOI: $10.1159 / 000506118$

(C) 2020 The Author(s). Published by S. Karger AG, Base www.karger.com/cnd

Nakatani et al.: Long-Term Effects of High-Dose Tolvaptan for Autosomal Dominant Polycystic Kidney Disease Patients

\section{Acknowledgements}

We are indebted to the nephrologists, the nursing staff, and the patient at Osaka City University Hospital for their collaboration.

\section{Statement of Ethics}

This report does not cite any experiments conducted with human or animal participants performed by any of the authors. Informed consent was obtained from the present patients for publication of relevant information in this case report. All procedures performed in studies involving human participants were in accordance with the ethical standards of the institutional committee at which the studies were conducted and with the 1964 Helsinki Declaration and its later amendments or comparable ethical standards.

\section{Disclosure Statement}

Eiji Ishimura received research grants from Otsuka Pharmaceutical Co., Ltd. (Tokyo, Japan). The other authors have no conflicts of interest to declare.

\section{Funding Sources}

There were no funding sources.

\section{Author Contributions}

S.N., Y.M., K.F., H.U., A.T., K.M., and E.I. were responsible for the clinical management of the patients and for preparing the draft version of the manuscript. M.E. and M.I. read and approved the manuscript. All authors participated in the writing of the manuscript and read and approved the final version.

\section{References}

1 Torres VE, Harris PC, Pirson Y. Autosomal dominant polycystic kidney disease. Lancet. 2007 Apr;369(9569):1287-301.

2 Hanafusa N, Nakai S, Iseki K, Tsubakihara Y. Japanese society for dialysis therapy renal data registry - a window through which we can view the details of Japanese dialysis population. Kidney Int Suppl (2011). 2015 Jun;5(1):15-22.

3 Torres VE, Harris PC. Strategies targeting cAMP signaling in the treatment of polycystic kidney disease. J Am Soc Nephrol. 2014 Jan;25(1):18-32.

4 Gattone VH 2nd, Wang X, Harris PC, Torres VE. Inhibition of renal cystic disease development and progression by a vasopressin V2 receptor antagonist. Nat Med. 2003 Oct;9(10):1323-6. 
5 Reif GA, Yamaguchi T, Nivens E, Fujiki H, Pinto CS, Wallace DP. Tolvaptan inhibits ERK-dependent cell proliferation, $\mathrm{Cl}^{-}$secretion, and in vitro cyst growth of human ADPKD cells stimulated by vasopressin. Am J Physiol Renal Physiol. 2011 Nov;301(5):F1005-13.

6 Torres VE, Chapman AB, Devuyst O, Gansevoort RT, Grantham JJ, Higashihara E, et al.; TEMPO 3:4 Trial Investigators. Tolvaptan in patients with autosomal dominant polycystic kidney disease. N Engl J Med. 2012 Dec;367(25):2407-18.

7 Torres VE, Chapman AB, Devuyst O, Gansevoort RT, Perrone RD, Koch G, et al.; REPRISE Trial Investigators. Tolvaptan in Later-Stage Autosomal Dominant Polycystic Kidney Disease. N Engl J Med. 2017 Nov;377(20):1930-42.

8 Edwards ME, Chebib FT, Irazabal MV, Ofstie TG, Bungum LA, Metzger AJ, et al. Long-Term Administration of Tolvaptan in Autosomal Dominant Polycystic Kidney Disease. Clin J Am Soc Nephrol. 2018 Aug;13(8):115361.

9 Horio M, Imai E, Yasuda Y, Watanabe T, Matsuo S. Modification of the CKD epidemiology collaboration (CKDEPI) equation for Japanese: accuracy and use for population estimates. Am J Kidney Dis. 2010 Jul;56(1):32-8.

10 Cockcroft DW, Gault MH. Prediction of creatinine clearance from serum creatinine. Nephron. 1976;16(1):3141.

11 Muto S, Kawano H, Higashihara E, Narita I, Ubara Y, Matsuzaki T, et al. The effect of tolvaptan on autosomal dominant polycystic kidney disease patients: a subgroup analysis of the Japanese patient subset from TEMPO 3:4 trial. Clin Exp Nephrol. 2015 Oct;19(5):867-77.

12 Irazabal MV, Rangel LJ, Bergstralh EJ, Osborn SL, Harmon AJ, Sundsbak JL, et al.; CRISP Investigators. Imaging classification of autosomal dominant polycystic kidney disease: a simple model for selecting patients for clinical trials. J Am Soc Nephrol. 2015 Jan;26(1):160-72.

13 Torres VE, Higashihara E, Devuyst 0, Chapman AB, Gansevoort RT, Grantham JJ, et al.; TEMPO 3:4 Trial Investigators. Effect of Tolvaptan in Autosomal Dominant Polycystic Kidney Disease by CKD Stage: Results from the TEMPO 3:4 Trial. Clin J Am Soc Nephrol. 2016 May;11(5):803-11.

14 Grantham JJ, Chapman AB, Torres VE. Volume progression in autosomal dominant polycystic kidney disease: the major factor determining clinical outcomes. Clin J Am Soc Nephrol. 2006 Jan;1(1):148-57.

15 Chebib FT, Perrone RD, Chapman AB, Dahl NK, Harris PC, Mrug M, et al. A Practical Guide for Treatment of Rapidly Progressive ADPKD with Tolvaptan. J Am Soc Nephrol. 2018 Oct;29(10):2458-70. 
Nakatani et al.: Long-Term Effects of High-Dose Tolvaptan for Autosomal Dominant

A

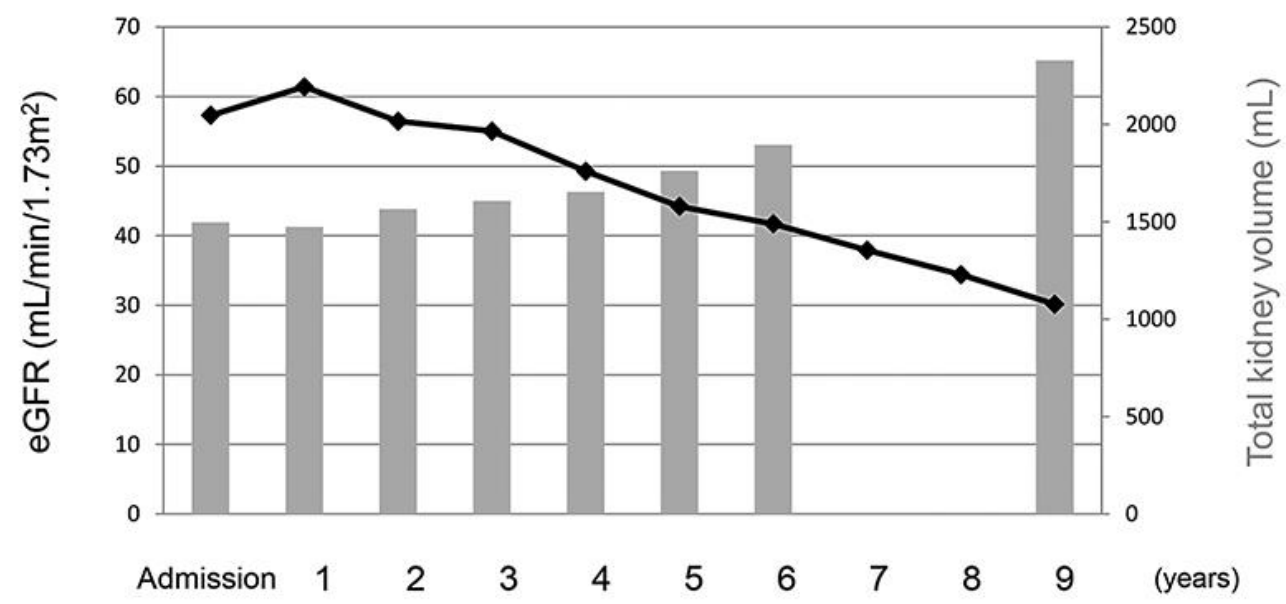

B

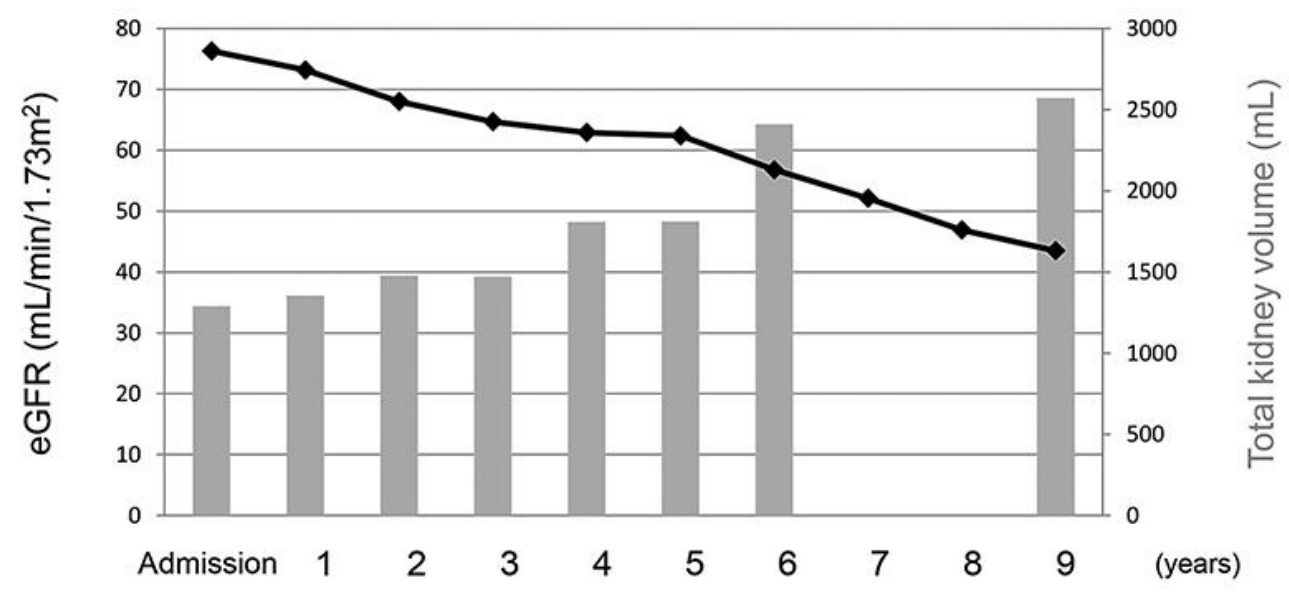

Fig. 1. Clinical courses of ADPKD patients who received tolvaptan therapy. A Case 1. B Case 2. Solid line: estimated glomerular filtration rate (eGFR); gray bar: total kidney volume. 


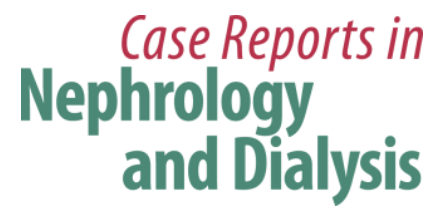

\begin{tabular}{l|l} 
Case Rep Nephrol Dial 2020;10:9-17 \\
\hline DOI: 10.1159/000506118 & $\begin{array}{l}\text { (c) 2020 The Author(s). Published by S. Karger AG, Basel } \\
\text { www.karger.com/cnd }\end{array}$ \\
\hline
\end{tabular}

Nakatani et al.: Long-Term Effects of High-Dose Tolvaptan for Autosomal Dominant Polycystic Kidney Disease Patients

Table 1. Laboratory examination findings prior to beginning tolvaptan treatment

\begin{tabular}{lll}
\hline & Case 1 & Case 2 \\
\hline Age, years & 36 & 29 \\
Body mass index, kg/m² & 22.0 & 28.4 \\
White blood cells, $\mu \mathrm{L}$ & 4,800 & 6,800 \\
Red blood cells, $\mu \mathrm{L}$ & 487 & 463 \\
Hemoglobin, g/dL & 14.5 & 13.9 \\
Total protein, g/dL & 6.9 & 7.2 \\
Albumin, g/dL & 4.5 & 4.6 \\
Blood urea nitrogen, mg/dL & 17 & 11 \\
Creatinine, mg/dL & 1.19 & 0.97 \\
Estimated glomerular filtration rate, & & \\
$\quad$ mL/min/1.73 m ${ }^{2}$ & 57.3 & 76.3 \\
\hline Estimated creatinine clearance, & & \\
$\quad$ mL/min & 78.1 & 125 \\
Uric acid, mg/dL & 5.5 & 7.4 \\
Aspartate transaminase, IU/L & 21 & 28 \\
Alanine transaminase, IU/L & 21 & 29 \\
Sodium, mEq/L & 145 & 143 \\
Potassium, mEq/L & 4.1 & 4.2 \\
Chloride, mEq/L & 105 & 106 \\
Urinary protein & $( \pm)$ & $(-)$ \\
Urinary occult blood & $(-)$ & $(-)$ \\
\hline
\end{tabular}

